\title{
Repetitive Transcranial Magnetic Stimulation Increases the Release of Dopamine in the Nucleus Accumbens Shell of Morphine-Sensitized Rats During Abstinence
}

\author{
A Erhardt', I Sillaber', T Welt', MB Müller', N Singewald² and ME Keck*,' \\ 'Max Planck Institute of Psychiatry, Munich, Germany; ${ }^{2}$ Department of Pharmacology and Toxicology, University of Innsbruck, Innsbruck, Austria
}

Recent studies in rodents have shown that withdrawal from chronic drug abuse is associated with a significant decrease in dopamine (DA) release in mesolimbic structures, especially in the shell region of the nucleus accumbens. Since the DA system is known to play an important role in reward processes, a withdrawal-associated impairment in mesolimbic DA-mediated transmission could possibly implicate reward deficit and thus enhance vulnerability to drug craving and relapse. We have previously demonstrated that acute repetitive transcranial magnetic stimulation (rTMS) has a modulatory effect on DA release in several areas of the rat brain, including dorsal striatum, hippocampus, and nucleus accumbens shell. In the present study, we investigated the possible use of rTMS as a tool in reestablishing the dysregulated DA secretion observed during withdrawal in morphine-sensitized male Sprague-Dawley rats. Using intracerebral microdialysis, we monitored the effects of acute rTMS $(20 \mathrm{~Hz})$ on the intra-accumbal release-patterns of DA in freely moving animals that were subjected to a morphine sensitization scheme for a period of 8 days. We provide first evidence that acute rTMS $(20 \mathrm{~Hz})$ is able to increase DA concentration in the shell region of the nucleus accumbens in both control animals and morphinesensitized rats during abstinence. The DA release in morphine-sensitized rats was significantly higher than in controls. rTMS, therefore, might gain a potential therapeutic role in the treatment of dysphoric and anhedonic states during drug withdrawal in humans. Neuropsychopharmacology (2004) 29, 2074-2080, advance online publication, 9 June 2004; doi:I 0. I038/sj.npp. I 300493

Keywords: rTMS; dopamine; morphine; drug withdrawal; craving; sensitization

\section{INTRODUCTION}

The mesolimbic dopaminergic system has been recognized to play a crucial role in the expression of natural consumatory behavior and its rewarding actions. Anatomically, it consists of the midbrain dopaminergic projection that originates in the ventral tegmental area (VTA, A10 cell group) and projects to structures closely associated with the limbic system, most prominently the nucleus accumbens shell region (ie nucleus accumbens septi, NAS) and the prefrontal cortex (Zahm, 2000). A wide range of studies, including electrical self-stimulation, cellular electrophysiology, and self-administration of drugs have indicated that the neurotransmitter dopamine (DA) participates in the processing of reward (Di Chiara, 1999; Spanagel and Weiss, 1999; Zahm, 2000). The release of DA in the mesolimbic system is supposed to mediate positive reinforcing effects

* Correspondence: ME Keck, Max Planck Institute of Psychiatry, Kraepelinstr. 2-10, D-80804 Munich, Germany, Tel: + 498930622 233, Fax: + 498930622 610, E-mail: keck@mpipsykl.mpg.de

Received 4 February 2004; revised 18 March 2004; accepted 20 April 2004

Online publication: 21 April 2004 at http://www.acnp.org/citations/ Npp42 I 0404053/default.pdf that ultimately shape behaviors for success accompanied by experience of pleasure, whereas disturbances in dopaminergic transmission could result in dysphoric unrewarding states (Wise, 1998). Not only natural rewards but also many drugs of abuse such as morphine and cocaine have the ability to increase the extracellular DA levels in the NAS after acute administration (Di Chiara and Imperato, 1986; Maisonneuve et al, 2001; Wise et al, 1995). In addition, chronic administration of addictive substances produces a number of adaptive changes in the central nervous system that lead to an increase, such as sensitization, or decrease, such as tolerance, of their behavioral effects (review: Nestler, 2002).

The neurobiological term 'sensitization' describes the phenomenon that repeated exposure to a drug such as morphine results in a progressive increase in its psychomotor stimulating and positive reinforcing effects. This phenomenon is causally related to the morphine-induced long-lasting hyper-reactivity of dopaminergic nerve terminals especially in the NAS (Cadoni and Di Chiara, 1999; Vanderschuren et al, 2001; Vanderschuren and Kalivas, 2000). Similarly, both tolerance and withdrawal appear to emerge from chronic reactive changes in dopaminergic neurotransmission, resulting in diminished effects of drugs 
of abuse (Johnson and Glick, 1993). In response to a longlasting overstimulation of dopaminergic neurons, the normal function of the system seems to become dependent on the presence of the exogenous substance. Drug abstinence, therefore, might result in understimulation of dopaminergic neurons followed by a relative lack of DA in the mesolimbic system, thus producing emotional withdrawal symptoms such as dysphoria and anhedonia in both animal models and humans (Acquas and Di Chiara, 1992; Ahtee et al, 1989; Volkow et al, 2002). So far, there is no satisfactory therapy for this anhedonic emotional state which has an enormous influence on the outcome of clinical treatment of addiction, leading to relapse in a large number of patients (Mattick et al, 2002).

rTMS is a noninvasive method for electrical stimulation of neurons in the cerebral cortex and is able to modify neuronal activity locally and in remote brain structures via transsynaptic connections (Nahas et al, 2001; Post and Keck, 2001). The neurobiological effects of this novel therapeutic tool in humans are not sufficiently explained so far, but it has repeatedly been reported that the mesolimbic dopaminergic system plays an important role in mediating the mood improvements in depressed patients (review: Padberg and Möller, 2003; Schlaepfer et al, 2003). Recently, we found an increase in DA release in NAS, striatum, and hippocampus in response to acute rTMS (Keck et al, 2002), which could be replicated by others (Kanno et al, 2004; Zangen and Hyodo, 2002). Coherent to this finding, rTMS treatment induces clinical improvement in patients with Parkinson's disease, which is known to have a substantial lack of DA in the mesostriatal system (review: Siebner and Rothwell, 2003). Accordingly, in healthy volunteers, rTMS of frontal brain regions increased striatal DA release (Strafella et al, 2001). Taken together, these findings point towards the importance of DA as one putative component underlying the clinical effects of rTMS. Considering these data, rTMS could be a useful therapeutic tool to modulate the altered DA release in the mesolimbic dopaminergic system during the withdrawal of drugs of abuse, and might thus be able to decrease the severity of accompanying clinical symptoms such as dysphoria and anhedonia. In the light of these data, we set out to investigate the effects of acute rTMS $(20 \mathrm{~Hz})$ on the DA release in morphine-sensitized Sprague-Dawley rats during morphine abstinence by using in vivo microdialysis.

\section{MATERIALS AND METHODS}

\section{Animals}

The animal studies were performed in accordance to NIH guidelines and the Guide for the Care and Use of Laboratory Animals of the Government of Bavaria, Germany. The experiments were carried out on adult male SpragueDawley rats (age of 6 weeks at arrival; $250 \pm 25 \mathrm{~g}$ body weight; $n=23)$. The animals were immediately separated after the delivery from the supplier (Charles River, Sulzfeld, Germany) and housed singly under standard laboratory conditions $(12: 12 \mathrm{~h}$ light/dark cycle with lights on at 0700 , $22 \pm 1{ }^{\circ} \mathrm{C}, 60 \%$ humidity, pelleted food and water ad libitum) at least 2 weeks before the experiment. Each animal was used for one experiment only.

\section{Drugs}

Morphine hydrochloride (Merck, Darmstadt) was dissolved in $0.9 \%$ saline and injected subcutaneously (s.c.) during a period of 1 week. The morphine sensitization injection scheme was adapted from Acquas and Di Chiara (1992). Rats were taken from their home cages, injected s.c. with morphine or $0.9 \%$ saline at a volume of $1 \mathrm{ml} / \mathrm{kg}$ body weight and returned immediately to the cage. The animals were injected twice daily (12-h interval) with a dose of $10 \mathrm{mg} / \mathrm{kg}$ during days $1-4$ and a dose of $15 \mathrm{mg} / \mathrm{kg}$ during days $5-7$. On day 8 , only one single injection of morphine $(10 \mathrm{mg} / \mathrm{kg})$ was administered to avoid withdrawal symptoms during the subsequent surgery. In the present study, low doses of morphine were used in order to avoid physical withdrawal symptoms after cessation. It has frequently been demonstrated in studies on behavioral sensitization that the effects of morphine on dopaminergic neurotransmission are mediated mainly through binding on $\mu$-opioid receptors when small doses are applied (Vezina et al, 1987; Vigano et al, 2003). We, therefore, chose a sensitization scheme using rather small amounts of morphine. Implication of other receptor types through higher morphine dosages would result in more severe morphine withdrawal symptoms and complex interactions with other neurotransmitter systems (Spanagel, 1995). On the last day of morphine injection, microdialysis surgery was performed. After a recovery period of $72 \mathrm{~h}$ rTMS was applied (day 11).

\section{Surgery}

Home-made, metal-free microdialysis probes were used for surgery (Keck et al, 2000b, 2002). The microdialysis probes consisted of a U-shaped microdialysis membrane (length of exposed dialysis membrane $1.5 \mathrm{~mm}$, molecular cutoff of $18 \mathrm{kDa}$; Hemophan TM, Gambro Dialysatoren, Hechingen, Germany), which was stabilized with a metal-free fiber and glued into the ends of two parallel fiberglass tubes (length: $20 \mathrm{~mm}$; inner diameter: $0.32 \mathrm{~mm}$; outer diameter: $0.45 \mathrm{~mm}$; Photonetics, Kehl, Germany). Before implantation, the microdialysis system was checked and filled with sterile standard Ringeŕs solution $\left(147.1 \mathrm{mM} / 1 \mathrm{Na}{ }^{+}, 2.25 \mathrm{mM} / \mathrm{l}\right.$ $\mathrm{Ca}^{+}, 4 \mathrm{mM} / 1 \mathrm{~K}^{+}, 155.6 \mathrm{mM} / \mathrm{l} \mathrm{Cl}^{-}, \mathrm{pH}$ 7.4; Fresenius, Bad Homburg, Germany). Afterwards, the microdialysis probes were stereotaxically implanted according to the atlas of Paxinos and Watson (1986) with their tips at the right NAS $(1.7 \mathrm{~mm}$ rostral to bregma, $1.0 \mathrm{~mm}$ lateral to midline, $8.0 \mathrm{~mm}$ beneath the surface of the skull) (Keck et al, 2002). The probes were fixed on the scull with dental cement. Animals were returned to the home cage and allowed to recover for 2 days.

\section{Repetitive Transcranial Magnetic Stimulation}

rTMS was applied exactly as described before (eg Czeh et al, 2002; Keck et al, 2000b, 2002; Müller et al, 2000). A commercially available stimulator (MagPro ${ }^{\text {TM }}$, DANTEC/ Medtronic, Skovlunde, Denmark) was used. The stimulus waveform was biphasic with a pulse width of $280 \mu$ s. The animals were stimulated with a round prototype coil (S 60, DANTEC; inner diameter: $6 \mathrm{~mm}$, outer diameter: $57 \mathrm{~mm} ; 21$ windings). Each animal received 300 stimuli resulting from 
six trains with a maximum magnetic induction field modulus of $4 \mathrm{~T}(120 \mathrm{~A} / \mu \mathrm{s} ; 130 \%$ of rat's motor threshold, determined as described previously) (Müller et al, 2000) at a rate of $20 \mathrm{~Hz}$ for $2.5 \mathrm{~s}$. These stimulation parameters were chosen according to the results of accurate magnetic resonance imaging (MRI)-based computer reconstructions as described before (Keck et al, 2000a, b, 2001). We chose a stimulation frequency of $20 \mathrm{~Hz}$ since we were able to demonstrate a clearcut stimulation of mesolimbic and mesostriatal dopaminergic systems when applying this frequency in previous studies (Keck et al, 2000b, 2002). Between trains there was an interval of $2.5 \mathrm{~min}$ for cooling to prevent overheating and possible damage of the stimulation coil. The coil was held in direct physical contact to the animal's head at the stimulation point. The stimulation point was set at the left frontal cortex, as determined according to the atlas of Greene (1963). Control rats were exposed to the acoustic artifact (ie clicking sound) with the stimulation coil held perpendicularly $10 \mathrm{~cm}$ above the head (sham stimulation).

A pilot study with unrestrained, unhandled, adult rats that had been subjected to the same rTMS protocol as in the present study showed clear, unspecific stress response as evidenced by locomotor hyperactivity and aggressive behavior (Keck et al, 2002). Several studies have shown that acute stress influences DA release in different parts of mesolimbic dopaminergic system (Piazza and Le Moal, 1997). Therefore, to exclude putative effects of the unspecific stress response on dopaminergic neurotransmission, rats were allowed to adapt to the rTMS artifact noise beginning at the age of 6 weeks and were subjected to a daily sham stimulation procedure. Rats adapted well to the rTMS procedure, as indicated by the fact that restraint of the animals was unnecessary for the time period needed to apply 300 stimuli. During the $2.5 \mathrm{~s}$ required for stimulation (one train), the head of the animals was slightly fixed with two fingers by the person who handled the rats during the training period in exactly the same way (Keck et al, 2000a). During the intertrain interval of $2.5 \mathrm{~min}$, rats could move freely. Sham stimulation was applied as described above with the control rats receiving six trains of sham stimulation. As handling is known to be able to influence intracerebral DA release (Feenstra et al, 1998), control rats were treated in exactly the same way as the rTMS animals during all parts of the experiment.

\section{Experimental Protocol}

After implantation of microdialysis probes, rats were housed singly in polycarbon cages $\left(23 \times 39 \times 36 \mathrm{~cm}^{3}\right)$ and handled daily until the experiment started. At 3 days after the surgery, microdialysis was performed. DA release was measured under basal conditions, during and after acute rTMS in 30-min microdialysates sampled from the right NAS in morphine-sensitized and control rats (morphine/ rTMS: $n=8$, morphine/sham: $n=7$, saline/rTMS: $n=8$ ) (Figure 2). First, the microdialysis probes were perfused with sterile Ringer's solution (flow rate: $2 \mu \mathrm{l} / \mathrm{min}$ ) via PEtubing connection to a microinfusion pump for $3 \mathrm{~h}$ without sampling. Previous studies in our group indicated that stable DA levels could be achieved after an adaptation time of $2 \mathrm{~h}$ (Keck et al, 2000b, 2002). Second, two basal samples were collected. During the third dialysis period, animals were subjected to rTMS, further three samples were collected afterwards (Figure 2). Samples were collected in Eppendorf tubes, containing $10 \mu \mathrm{l} 0.1 \mathrm{~N} \mathrm{HClO}_{4}$, transferred on dry ice and stored at $-80^{\circ} \mathrm{C}$ until DA measurement.

\section{Dopamine Radioimmunoassay}

Samples were stored at $-80^{\circ} \mathrm{C}$ until biochemical analysis. DA released in the superfusate was determined by a radioenzymatic assay as previously described (Singewald and Philippu, 1993). This assay involves COMT-catalyzed $O$-methylation using $\left[{ }^{3} \mathrm{H}\right] S$-adenosylmethionine as methyl donor and separation of the resulting $\left[{ }^{3} \mathrm{H}\right]$ normetanephrine by TLC. The sensitivity of the assay was $0.5 \mathrm{pg}$ DA/sample.

\section{Histological Verification of Probe Placement}

Animals were killed with an overdose of halothane at the end of the experiments. Subsequently, brains were removed, frozen in prechilled n-methylbutane on dry ice, and stored at $-80^{\circ} \mathrm{C}$. For exact histological verification of the placement of the microdialysis probes, brains were sectioned in a cryostat, and $25-\mu \mathrm{m}$ frontal sections were stained with cresyl violet (Figure 1).

\section{Statistics}

Statistical analysis was performed with a statistical software package (Statistica 5.0, Statsoft, Tusla, USA). Two-way analysis of variance (ANOVA) (group $\times$ time) for repeated measures was applied to the data obtained from the assays of DA. The DA levels from microdialysis experiments are expressed as a percentage of averaged baseline values. Results showing significant overall changes were subjected to post hoc Fisher-LSD test comparison. $P<0.05$ was considered statistically significant. Data are presented as means \pm SEM.

\section{RESULTS}

Experimental animals were included in the statistical analysis only if (1) the microdialysis probes were found to be localized within the respective brain area (Figure 1); and (2) post-mortem visual examination failed to show any organ pathology. In total, $82 \%$ of microdialysis probes were

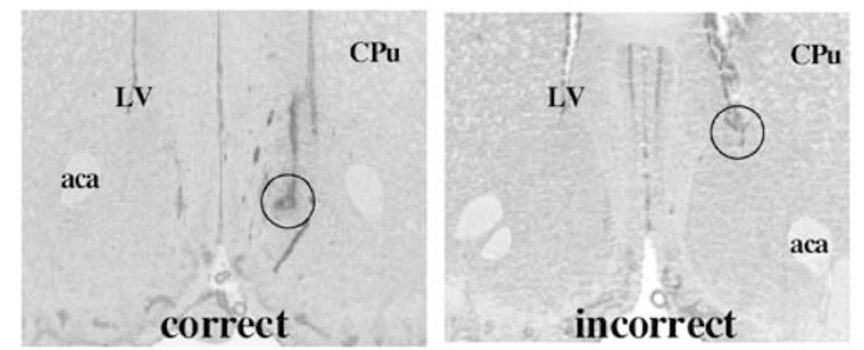

Figure I Histological placement of the tip of microdialysis probe in the nucleus accumbens shell. Aca: anterior commissure anterior part; CPu: caudate putamen (striatum); LV: lateral ventricle. 
implanted correctly (total number of animals: $n=28$; correctly localized probes: $n=23$ ).

\section{Extracellular Basal Levels}

The basal levels of extracellular DA in the nucleus accumbens shell of male Sprague-Dawley rats were not significantly different among the various groups (post hoc test: Fisher's LSD). Basal levels (mean of two 30-min sampling periods \pm SEM), expressed as pmol/60 $\mu \mathrm{l}$, were the following: group 1 (morphine/rTMS, $n=8$ ) $1.61 \pm 0.07$; group 2 (morphine/sham, $n=7$ ) 1.74 \pm 0.14 ; group 3 (saline/rTMS, $n=8$ ) $1.68 \pm 0.11$.

\section{Effects of Acute rTMS on DA Levels}

Microdialysate levels of DA showed a significant increase in response to rTMS in both groups, morphine-sensitized $(n=8)$ and saline-injected $(n=8)$ rats (two-way ANOVA for repeated measures). In addition, there was a significant sensitization effect with respect to DA release in the NAS of morphine-sensitized rats. In contrast to sham-stimulated rats $(n=7)$, there was a significant increase in intraaccumbal release of DA of morphine-sensitized animals during the third, fourth, fifth, and sixth microdialysis interval as confirmed by two-way ANOVA followed by post hoc Fisher's LSD test (Figure 2). Statistical analysis revealed a significant effect of the factor group $\left(\mathrm{F}_{2,20}=9.31\right.$, $P<0.01)$, the factor time $\left(\mathrm{F}_{1,140}=10.72 ; P<0.01\right)$, and the factor group $\times$ time $\left(\mathrm{F}_{14,140}=3.58 ; P<0.01\right)$. The maximum increase in DA release was the following: group 1 (morphine/rTMS): to $2.56 \pm 0.18$ during the fifth microdialysis interval; group 3 (saline/rTMS): to $2.35 \pm 0.15$ during the fifth microdialysis interval. The DA levels in group 2 (morphine/sham) remained stable during and after acute rTMS.

\section{DISCUSSION}

The results obtained provide first evidence that acute left frontal rTMS $(20 \mathrm{~Hz})$ is able to modulate dopaminergic

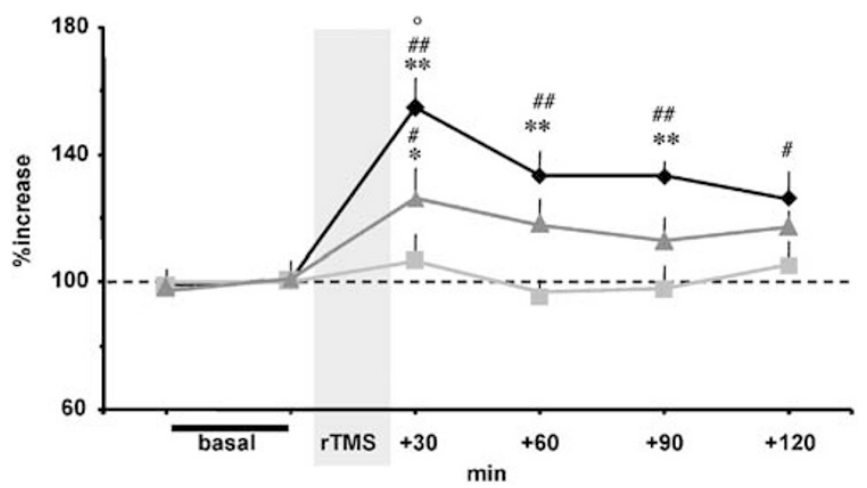

Figure 2 Effects of rTMS $(20 \mathrm{~Hz}$, six trains, 300 stimuli) on the dopamine content of 30-min dialysates collected consecutively from the NAS in morphine-sensitized (rhombs, morphine/rTMS, $n=8$ ) and saline-treated rats (triangles, saline/rTMS, $n=8$ ) vs sham-stimulated, morphine-sensitized conscious Sprague-Dawley rats. Data are expressed as percentage of averaged baseline values \pm SEM. $* P<0.05$, ** $P<0.01$ vs sham stimulation; ${ }^{\#} P<0.05,{ }^{\# \#} P<0.0$ I vs basal; ${ }^{\circ} P<0.05$ vs saline (post hoc Fisher's LSD test). neurotransmission in the NAS in both morphine-sensitized rats during morphine abstinence and saline-treated animals, herewith confirming previous findings that rTMS increases DA release in NAS, hippocampus and striatum (Keck et al, 2002; Zangen and Hyodo, 2002; Kanno et al, 2004). The rTMS-induced DA release in the morphinetreated group was approximately $30 \%$ higher than in the saline-treated group. This observation indicates a clear sensitization effect of long-term morphine treatment with subsequent neuronal alterations in the mesolimbic system (review: Koob, 2001).

\section{rTMS in Rat Studies}

Although in most cases not anatomically precise (Herwig et al, 2001), rTMS effects in clinical studies most probably relate to frontal forebrain stimulation (review: eg Padberg and Möller, 2003; Schlaepfer et al, 2003). Consequently, to reliably investigate the underlying neurobiological effects in animal models, the adoption of equivalent stimulation conditions is indispensable. It is worth noting that magnetic stimulation of rodent brains is not diffuse by necessity (Siebner and Rothwell, 2003). One way to address these problems is to calculate the spatial distribution of current density induced in both the rat and human brain and to adjust the stimulation parameters accordingly. The coil and stimulation parameters used in the studies conducted at our laboratory were selected according to an exact characterization of the conductive phenomena elicited by rTMS in both human and rat brain (eg Keck et al, 2000a, b, 2001, 2002; Czeh et al, 2002). This enabled us to accurately adapt the experimental setup in order to achieve a stimulation pattern that is analogous to the one used in patients during standard clinical treatment. The results of the above procedure show that our experimental setup enables us to obtain a stimulation pattern which exhibits a definite peak in the left frontal brain region as desired (eg Keck et al, 2000a, b, 2001). It is, therefore, justified to interpret subsequently collected data as related to selective stimulation of this brain area (for a review see Keck, 2003).

\section{Neurobiological Changes in Chronic Opioid Use}

Pharmacological studies have shown that $\mu$-opioid-receptors in the VTA, from where mesolimbic DA cells project to the nucleus accumbens, mediate the rewarding and reinforcing effects of morphine leading to addiction (Spanagel et al, 1993). Stimulation of $\mu$-opioid receptors in the VTA enhances mesolimbic dopaminergic neurotransmission, presumably by inhibition of gamma-amino-butyricacid-(GABA)ergic interneurons, thereby dysinhibiting mesolimbic dopaminergic neurons and increasing both somatodendritic and axonal DA release (Klitenick et al, 1992; Wise et al, 1995). It has repeatedly been demonstrated in studies on behavioral sensitization that the effects of morphine on dopaminergic neurotransmission are mediated mainly through binding on this receptor type when small doses are applied (Vezina et al, 1987; Vigano et al, 2003). We, therefore, chose a sensitization scheme using rather small amounts of morphine. The effects of sensitization are important for the initial increase of the rewarding effects of drugs of abuse, presumably leading to 
an enhanced intake of the drug and the development of addiction (De Vries and Shippenberg, 2002; Wise, 1998). Pre- and postsynaptic changes in sensitized animals have been described, such as local downregulation of DA-type-2(D2)-autoreceptors and an increased sensitivity of postsynaptic D1-receptors (Berke and Hyman, 2000; White, 1996). The sensitization effect observed in our study verifies that the morphine injection scheme induced neurobiological changes in dopaminergic neurotransmission and that rTMS can effectively modulate the DA release under this condition, which is comparable, at last in part, to that found in addicted humans during abstinence (review: Nestler, 2002).

Currently available therapies for the treatment of opioid addiction are mainly based on replacing a short acting opioid drug with a long acting one, such as methadone, with continuous dose reduction. By use of the latter approach, the rate of success is around $30 \%$ (review: Mattick et al, 2002). The somatic withdrawal symptoms are less dramatic for the majority of addicted patients returning to control values within several days. The appearance of longer lasting anhedonic and dysphoric mood states during acute withdrawal or within the state of craving, however, is considered to be more important for the risk of relapse (review: Mattick et al, 2002). The different duration of the somatic and psychiatric symptoms underlines the assumption that they are generated in two different neuroanatomical systems: the former are partly elicited from alterations in the periaqueductal gray area including various transmitter systems (Wise, 1996), whereas the latter are likely to result from neurobiological changes in dopaminergic mesolimbic neurotransmission (Koob, 2001). Accordingly, electrophysiological analysis of mesolimbic dopaminergic neurons and microdialysis studies in rats during opioid withdrawal revealed a lasting tonic reduction of the neuronal firing rate and DA release after chronic morphine administration (eg Acquas and Di Chiara, 1992; Diana et al, 1999). This finding is consistent with the results from intracranial selfstimulation studies (ICSS), showing an increased rewarding threshold during drug withdrawal (Nazzaro et al, 1981).

In the present experiment, we failed to detect decreased extracellular levels of DA in the NAS $72 \mathrm{~h}$ after the last morphine injection. It is possible that the intermittent morphine injection scheme applied was not sufficient to induce a reduction of extracellular concentrations of DA in the NAS after cessation. In studies using higher doses of morphine, a clearcut decline in NAS DA release has repeatedly been reported (eg Acquas and Di Chiara, 1992; Diana et al, 1999). In our study, however, a morphine injection scheme with low concentrations was applied because we aimed at avoiding the occurrence of physical withdrawal symptoms that are associated with hypothalamic-pituitary-adrenocortical-(HPA)-system activation (Piazza and Le Moal, 1997). This was done in order to investigate the modulatory effects of $20 \mathrm{~Hz}$ rTMS on mesolimbic dopaminergic neurons without its possibly confounding effects on HPA system activity (Keck et al, 2000a, 2001; Hedges et al, 2002; Czeh et al, 2002), which, in turn, would influence intra-accumbal DA release. In any case, as has previously been shown by Acquas and Di Chiara (1992), we were able to induce robust neurobiological adaptations as reflected by dopaminergic sensitization, a phenomenon that has repeatedly been suggested to play an important role in the etiology of addiction (review: Robinson and Berridge, 2001).

\section{Mesolimbic Dopaminergic System as a Therapeutic Target for rTMS?}

Previous studies dealing with the neurobiological effects of $20 \mathrm{~Hz}$ rTMS showed that the serotonergic and noradrenergic neurotransmission in hippocampus, striatum, and NAS remained unchanged after acute left frontal rTMS (Keck et al, 2000b, 2002). Although preliminary, positive clinical effects in the treatment of Parkinson's disease with rTMS seem to point towards the significant role of dopaminergic neurotransmission as a mechanism of rTMS action (review: Siebner and Rothwell, 2003; Tsuji and Akamatsu, 2003). Our report of an acute occurrence of psychotic symptoms in response to rTMS is also in favor of an rTMS-induced increase in dopaminergic activity (Zwanzger et al, 2002). Accordingly, rTMS was reported to have better effects in nonpsychotic as compared to psychotic major depression (Grunhaus et al, 2000). In the latter condition, a dopaminergic hyperactivity is thought to play a crucial role. We therefore assumed that the clinical effects of rTMS might be mediated in part through interactions with the mesolimbic and mesostriatal dopaminergic systems (Keck et al, 2000b, 2002). A multitude of stimulation frequencies being possible, it is of interest to note that in studies conducted at other laboratories, rTMS frequencies of 2 and $25 \mathrm{~Hz}$ were also able to increase dopaminergic neurotransmission (Zangen and Hyodo, 2002; Kanno et al, 2004).

A possible mechanism for the rTMS-induced increase in DA release is the stimulation of excitatory projections from the prefrontal cortex. MRI-based, computer-assisted characterizations of the electric current density induced by left frontal rTMS show a maximal current induction within frontal cortical regions (Keck et al, 2000a, b, 2001). This brain area belongs to the mesocorticolimbic system and sends dense afferents to the VTA, which in turn projects to the NAS and hippocampus (Sesack, 2001; Zahm, 2000). In support of this, an rTMS-induced increase in dopaminergic neurotransmission in striatal regions has been demonstrated in healthy volunteers by use of positron emission tomography (Strafella et al, 2001).

rTMS could furthermore modulate mesolimbic DA concentrations through stimulation of glutamatergic projections from the prefrontal cortex to the NAS (You et al, 1998), which are able to increase DA release by a local effect of glutamate on adjacent dopaminergic nerve terminals from the VTA (Cheramy et al, 1998; Taber and Fibiger, 1995). Additional rTMS-induced neurobiological changes, such as an increase in gene expression of the neuropeptide cholecystokinin (CCK), may contribute to the effects reported, since CCK, acting as a neuromodulator, increases the firing rate of dopaminergic neurons (Crawley and Corwin, 1994; Müller et al, 2000).

In conclusion, the present results suggest that rTMS of left frontal brain regions may be an effective therapeutic tool in the treatment of acute withdrawal symptoms. Preliminary evidence from a study where $20 \mathrm{~Hz}$ rTMS was able to reduce cigarette smoking is in support of this assumption (Eichhammer et al, 2003). It has to be noted, however, that 
results obtained in an animal model assessing opioid effects cannot necessarily be generalized to other psychotropic drugs. Detailed clinical studies must follow to test our hypothesis.

\section{ACKNOWLEDGEMENTS}

This project is supported by the German Federal Research Ministry within the promotional emphasis 'Competence Nets in Medicine' (Kompetenznetz Depression \& Suizidalität; subproject $4.5, \mathrm{MEK}$ ) and the Bavarian Research Ministry (MEK). We thank M Rücker and U Genning for expert technical assistance. DANTEC/Medtronic, Skovlunde, Denmark, generously provided the stimulation device.

\section{REFERENCES}

Acquas E, Di Chiara G (1992). Depression of mesolimbic dopamine transmission and sensitization to morphine during opiate abstinence. J Neurochem 58: 1620-1625.

Ahtee L, Attila LM, Carlson KR, Haikala H (1989). Changes in brain monoamine metabolism during withdrawal from chronic oral self-administration of morphine and in response to a morphine challenge in the withdrawn state. J Pharmacol Exp Ther 249: 303-310.

Berke JD, Hyman SE (2000). Addiction, dopamine, and the molecular mechanisms of memory. Neuron 25: 515-532.

Cadoni C, Di Chiara G (1999). Reciprocal changes in dopamine responsiveness in the nucleus accumbens shell and core and in the dorsal caudate-putamen in rats sensitized to morphine. Neuroscience 90: 447-455.

Cheramy A, L'Hirondel M, Godeheu G, Artaud F, Glowinski J (1998). Direct and indirect presynaptic control of dopamine release by excitatory amino acids. Amino Acids 14: 63-68.

Crawley JN, Corwin RL (1994). Biological actions of cholecystokinin. Peptides 15: 731-755.

Czeh B, Welt T, Fischer AK, Erhardt A, Schmitt W, Muller MB et al (2002). Chronic psychosocial stress and concomitant repetitive transcranial magnetic stimulation: effects on stress hormone levels and adult hippocampal neurogenesis. Biol Psychiatry 52: 1057-1065.

De Vries TJ, Shippenberg TS (2002). Neural systems underlying opiate addiction. J Neurosci 22: 3321-3325.

Diana M, Muntoni AL, Pistis M, Melis M, Gessa GL (1999). Lasting reduction in mesolimbic dopamine neuronal activity after morphine withdrawal. Eur J Neurosci 11: 1037-1041.

Di Chiara G (1999). Drug addiction as dopamine-dependent associative learning disorder. Eur J Pharmacol 375: 13-30.

Di Chiara G, Imperato A (1986). Preferential stimulation of dopamine release in the nucleus accumbens by opiates, alcohol, and barbiturates: studies with transcerebral dialysis in freely moving rats. Ann NY Acad Sci 473: 367-381.

Eichhammer P, Johann M, Kharraz A, Binder H, Pittrow D, Wodarz $\mathrm{N}$ et al (2003). High-frequency repetitive transcranial magnetic stimulation decreases cigarette smoking. J Clin Psychiatry 64: 951-953.

Feenstra MGP, Botterblom MHA, van Uum JFM (1998). Local activation of metabotropic glutamate receptors inhibits the handling-induced increased release of dopamine in the nucleus accumbens but not that of dopamine or noradrenaline in the prefrontal cortex: comparison with inhibition of ionotropic receptors. J Neurochem 70: 1104-1113.

Greene EC (1963). Anatomy of the Rat. Hafner Publishing Company: New York and London.
Grunhaus L, Dannon PN, Schreiber S, Dolberg OH, Amiaz R, Ziv R et al (2000). Repetitive transcranial magnetic stimulation is as effective as electroconvulsive therapy in the treatment of nondelusional major depressive disorder: an open study. Biol Psychiatry 47: 314-324.

Hedges DW, Salyer DL, Higginbotham BJ, Lund TD, Hellewell JL, Ferguson D et al (2002). Transcranial magnetic stimulation (TMS) effects on testosterone, prolactin, and corticosterone in adult male rats. Biol Psychiatry 51: 417-421.

Herwig U, Padberg F, Unger J, Spitzer M, Schönfeldt-Lecuona C (2001). Transcranial magnetic stimulation in therapy studies: examination of the reliability of "standard" coil positioning by neuronavigation. Biol Psychiatry 50: 58-61.

Johnson DW, Glick SD (1993). Dopamine release and metabolism in nucleus accumbens and striatum of morphine-tolerant and nontolerant rats. Pharmacol Biochem Behav 46: 341-347.

Kanno M, Matsumoto M, Togashi H, Yoshioka M, Mano Y (2004). Effects of acute repetitive transcranial magnetic stimulation on dopamine release in the rat dorsolateral striatum. J Neurol Sci 217: 73-81.

Keck ME (2003). rTMS as treatment strategy in psychiatric disorders-neurobiological concepts. Clin Neurophysiol 56(Suppl): 100-116.

Keck ME, Engelmann M, Müller MB, Henniger MSH, Hermann B, Rupprecht $\mathrm{R}$ et al (2000a). Repetitive transcranial magnetic stimulation induces active coping strategies and attenuates the neuroendocrine stress response in rats. J Psychiatr Res 34: 265-276.

Keck ME, Sillaber I, Ebner K, Welt T, Toschi N, Kaehler ST et al (2000b). Acute transcranial magnetic stimulation of frontal brain regions selectively modulates the release of vasopressin, biogenic amines and amino acids in the rat brain. Eur J Neurosci 12: 3713-3720.

Keck ME, Welt T, Müller MB, Erhardt A, Ohl F, Toschi N et al (2002). Repetitive transcranial magnetic stimulation increases the release of dopamine in the mesolimbic and mesostriatal system. Neuropharmacology 43: 101-109.

Keck ME, Welt T, Post A, Müller AB, Toschi N, Wigger A et al (2001). Neuroendocrine and behavioral effects of repetitive transcranial magnetic stimulation in a psychopathological animal model are suggestive of antidepressant-like effects. Neuropsychopharmacology 24: 337-349.

Klitenick MA, DeWitte P, Kalivas PW (1992). Regulation of somatodendritic dopamine release in the ventral tegmental area by opioids and GABA: an in vivo microdialysis study. J Neurosci 12: $2623-2632$.

Koob GF (2001). Drug addiction, reward dysregulation and allostasis. Br J Pharmacol 134: 195.

Maisonneuve IM, Warner LM, Glick SD (2001). Biphasic doserelated effects of morphine on dopamine release. Drug Alcohol Depend 65: 55-63.

Mattick RP, Breen C, Kimber J, Davoli M (2002). Methadone maintenance therapy versus no opioid replacement therapy for opioid dependence. Cochrane Database Syst Rev 4: 1-12.

Müller MB, Toschi N, Kresse AE, Post A, Keck ME (2000). Long-term repetitive transcranial magnetic stimulation increases the expression of brain-derived neurotrophic factor and cholecystokinin mRNA, but not neuropeptide tyrosine mRNA in specific areas of rat brain. Neuropsychopharmacology 23: 205-215.

Nahas Z, Lomarev M, Roberts DR, Shastri A, Lorberbaum JP, Teneback C et al (2001). Unilateral left prefrontal transcranial magnetic stimulation (TMS) produces intensity-dependent bilateral effects as measured by interleaved BOLD fMRI. Biol Psychiatry 50: 712-720.

Nazzaro JM, Seeger TF, Gardner EL (1981). Morphine differentially affects ventral tegmental and substantia nigra brain reward thresholds. Pharmacol Biochem Behav 14: 325-331. 
Nestler EJ (2002). From neurobiology to treatment: progress against addiction. Nat Neurosci 5: 1076-1079.

Padberg F, Möller HJ (2003). Repetitive transcranial magnetic stimulation-does it have potential in the treatment of depression? Cns Drugs 17: 383-403.

Paxinos G, Watson C (1986). The Rat Brain in Stereotaxic Coordinates. Academic Press: Sydney.

Piazza PV, Le Moal M (1997). Glucocorticoids as a biological substrate of reward: physiological pathophysiological implications. Brain Res Rev 25: 359-372.

Post A, Keck ME (2001). Transcranial magnetic stimulation as a therapeutic tool in psychiatry: what do we know about the neurobiological mechanisms? J Psychiatr Res 35: 193-215.

Robinson TE, Berridge KC (2001). Incentive-sensitization and addiction. Addiction 96: 103-114.

Schlaepfer TE, Kosel M, Nemeroff CB (2003). Efficacy of repetitive transcranial magnetic stimulation (rTMS) in the treatment of affective disorders. Neuropsychopharmacology 28: 201-205.

Sesack SR (2001). Prefrontal cortical projections to the nucleus accumbens, amygdala, and ventral tegmental area: multiple substrates for prefrontal regulation of ventral striatal function. Biol Psychiatry 49: 9S.

Siebner HR, Rothwell J (2003). Transcranial magnetic stimulation: new insights into representational cortical plasticity. Exp Brain Res 148: 1-16.

Singewald N, Philippu A (1993). Catecholamine release in the locus coeruleus is modified by experimentally induced changes in haemodynamics. Naunyn Schmiedebergs Arch Pharmacol 347: 21-27.

Spanagel R (1995). Modulation of drug-induced sensitization processes by endogenous opioid systems. Behav Brain Res 70: 37-49.

Spanagel R, Almeida OF, Shippenberg TS (1993). Long lasting changes in morphine-induced mesolimbic dopamine release after chronic morphine exposure. Synapse 14: 243-245.

Spanagel R, Weiss F (1999). The dopamine hypothesis of reward: past and current status. Trends Neurosci 22: 521-527.

Strafella AP, Paus T, Barrett J, Dagher A (2001). Repetitive transcranial magnetic stimulation of the human prefrontal cortex induces dopamine release in the caudate nucleus. J Neurosci 21(RC 157): 1-4.

Tsuji S, Akamatsu N (2003). Does transcranial magnetic stimulation improve the motor symptoms of Parkinson disease? J Neurol 250(Suppl 3): 47-50.

Taber MT, Fibiger HC (1995). Electrical-stimulation of the prefrontal cortex increases dopamine release in the nucleus- accumbens of the rat-modulation by metabotropic glutamate receptors. J Neurosci 15: 3896-3904.

Vanderschuren LJ, De Vries TJ, Wardeh G, Hogenboom FA, Schoffelmeer AN (2001). A single exposure to morphine induces long-lasting behavioural and neurochemical sensitization in rats. Eur J Neurosci 14: 1533-1538.

Vanderschuren LJ, Kalivas PW (2000). Alterations in dopaminergic and glutamatergic transmission in the induction and expression of behavioral sensitization: a critical review of preclinical studies. Psychopharmacology 151: 99-120.

Vezina P, Kalivas PW, Stewart J (1987). Sensitization occurs to the locomotor effects of morphine and the specific mu opioid receptor agonist, DAGO, administered repeatedly to the ventral tegmental area but not to the nucleus accumbens. Brain Res 417: $51-58$.

Vigano D, Rubino T, Di Chiara G, Ascari I, Massi P, Parolaro D (2003). Mu opioid receptor signaling in morphine sensitization. Neuroscience 117: 921-929.

Volkow ND, Fowler JS, Wang GJ, Goldstein RZ (2002). Role of dopamine, the frontal cortex and memory circuits in drug addiction: insight from imaging studies. Neurobiol Learn Mem 78: 610-624.

White FJ (1996). Synaptic regulation of mesocorticolimbic dopamine neurons. Annu Rev Neurosci 19: 405-436.

Wise RA (1996). Addictive drugs and brain stimulation reward. Annu Rev Neurosci 19: 319-340.

Wise RA (1998). Drug-activation of brain reward pathways. Drug Alcohol Depend 51: 13-22.

Wise RA, Leone P, Rivest R, Leeb K (1995). Elevations of nucleus accumbens dopamine and DOPAC levels during intravenous heroin self-administration. Synapse 21: 140-148.

You ZB, Tzschentke TM, Brodin E, Wise RA (1998). Electrical stimulation of the prefrontal cortex increases cholecystokinin, glutamate, and dopamine release in the nucleus accumbens: an in vivo microdialysis study in freely moving rats. J Neurosci 18: 6492-6500.

Zahm DS (2000). An integrative neuroanatomical perspective on some subcortical substrates of adaptive responding with emphasis on the nucleus accumbens. Neurosci Biobehav Rev 24: 85-105.

Zangen A, Hyodo K (2002). Transcranial magnetic stimulation induces increases in extracellular levels of dopamine and glutamate in the nucleus accumbens. Neuroreport 13: 2401-2405. Zwanzger P, Ella R, Keck ME, Rupprecht R, Padberg F (2002). Occurrence of delusions during repetitive transcranial magnetic stimulation (rTMS) in major depression. Biol Psychiatry 51: 602-603. 